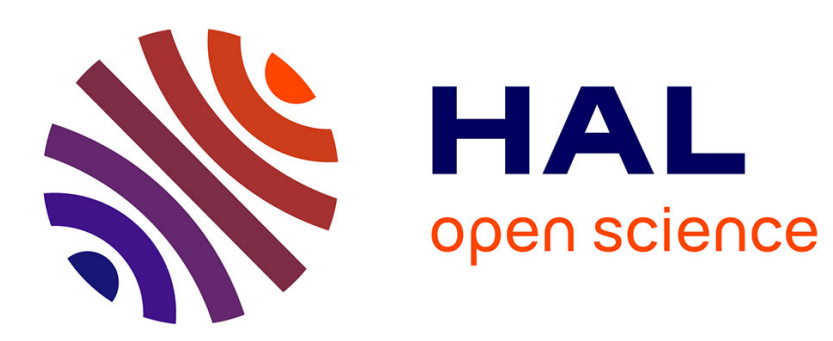

\title{
Cycling of implantation step and remote plasma process step for nitride spacer etching applications.
}

Nicolas Loubet, Cecile Jenny, Camille Petit-Etienne, Erwine Pargon

\section{To cite this version:}

Nicolas Loubet, Cecile Jenny, Camille Petit-Etienne, Erwine Pargon. Cycling of implantation step and remote plasma process step for nitride spacer etching applications.. Proceedings of the SPIE, 2020, 11329, pp.16. 10.1117/12.2551888 . hal-02912743

\section{HAL Id: hal-02912743 \\ https://hal.univ-grenoble-alpes.fr/hal-02912743}

Submitted on 19 Nov 2020

HAL is a multi-disciplinary open access archive for the deposit and dissemination of scientific research documents, whether they are published or not. The documents may come from teaching and research institutions in France or abroad, or from public or private research centers.
L'archive ouverte pluridisciplinaire HAL, est destinée au dépôt et à la diffusion de documents scientifiques de niveau recherche, publiés ou non, émanant des établissements d'enseignement et de recherche français ou étrangers, des laboratoires publics ou privés. 


\title{
Cycling of implantation step and remote plasma process step for nitride spacer etching applications.
}

\author{
Nicolas Alexandre Loubet ${ }^{\mathrm{a}, \mathrm{b}}$, Cécile Jenny $^{\mathrm{a}}$, Camille Petit-Etienne ${ }^{\mathrm{b}}$, Erwine Pargon ${ }^{\mathrm{b}}$ \\ ${ }^{a}$ STMicroelectronics, 850 rue Jean Monnet, 38926 Crolles Cedex, France ${ }^{\mathrm{b}}$ CNRS-LTM, UMR 5129, \\ Université Grenoble Alpes, CEA-Leti Minatec 17 rue des Martyrs, F-38054 Grenoble Cedex, \\ France.
}

\begin{abstract}
The etching of silicon nitride spacers is one of the most challenging steps of transistor fabrication. It requires anisotropy to preserve the sidewalls and a high etch selectivity over the underlying substrate to achieve a high surface quality. Recently, an interesting approach using a two step-process was proposed for the etching of silicon nitride spacers with high anisotropy and minimal induced damage [1]. The first step uses an $\mathrm{H}_{2}$ implantation to selectively modify the horizontal $\mathrm{SiN}$ surfaces over the vertical ones, while the second step selectively removes the modified layer either via HF exposure or via a remote plasma (RP). This paper explores a new route to implement those two steps in a cycling process achieved in the same plasma reactor chamber. The reactor has the capability to produce both a capacitive plasma discharge (CCP) for the implantation step and a remote discharge for the removal step. This study demonstrates that the remote plasma process, whose etching mechanisms are driven by reactive neutrals, is highly sensitive to the material surface state and consequently an incubation time exists before the etching starts when exposed to neutrals. The modifications induced by the first implantation step shortens the incubation time offering a process window with infinite etch selectivity between horizontal implanted and vertical non-implanted surfaces. Based on this understanding a two-step cycling process was developed and applied successfully to the etching of $\mathrm{Si}_{3} \mathrm{~N}_{4}$ spacer patterns for imager applications.
\end{abstract}

Keywords: SiN spacers etching, smart-etch concept, two-step cycling process, remote plasma.

\section{INTRODUCTION}

Spacers are key component of the transistor and the etching can modify the transistor performances as it determines the alignment of the source and the drain. Spacer etching is especially challenging, as it requires anisotropic spacer profile with minimal lateral consumption as well as high selectivity over the underlying Si. Those features can be difficult to achieve with conventional inductively coupled plasma (ICP) technology. Some integration requires the use of plasma enhanced chemical vapor deposited (PECVD) nitride film as a spacer material, instead of the conventional low pressure chemical vapor deposited (LPCVD) SiN. Because of their high hydrogen content and low density, PECVD SiN material is less plasma resistant than LPCVD SiN, Consequently, the high ion flux and energy of conventional ICP fluorocarboned process can lead to severe faceting and uncontrolled profiles as well as some silicon recess, as shown in Fig. 1.

a)

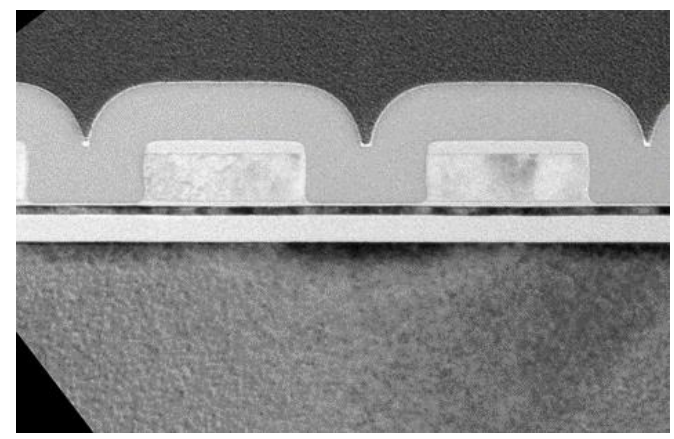

b)

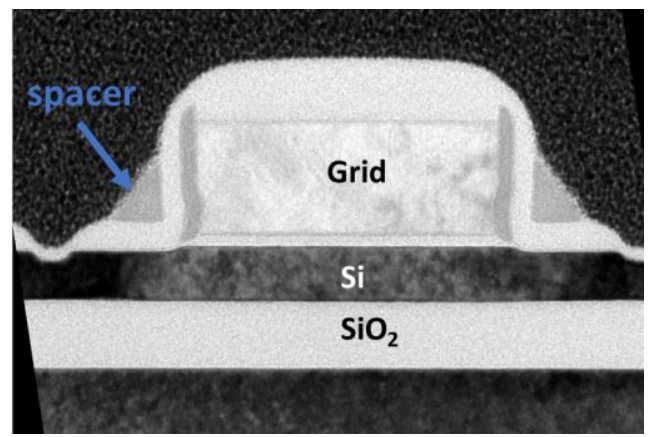

Figure 1. TEM picture of PECVD Silicon nitride a) before and b) after the conventional etch process used for LPCVD spacer etching at STMicroelectronics. 
An alternative approach proposed few years ago showed promising capabilities in terms of selectivity and anisotropy for silicon nitride spacer etching process [1]. This process called "smart etch" thereafter relies on two sequential steps. As shown in figure 2, in the first step, the layer is anisotropically modified by a light ion implantation (typically $\mathrm{H}_{2}$ or $\mathrm{He}$ ) achieved either in an ICP or a CCP reactor. The thickness of the modified layer is driven by the ion flux and energy [1] Anisotropy is achieved thanks to the ion directionality: the horizontal surfaces will be modified by the ion flux while the vertical surfaces will remain unmodified (considering the ion angular distribution negligible). The modified layer is then removed selectively over the non-modified layer and the underlying material using chemical etching based on either wet solution or downstream plasma [2][3]. The concept was initially developed using two different tools for the implantation and the removal steps [1]. More recently, our group showed that those two steps can be carried out in the same reactor chamber [4]. This reactor is a prototype offering two operating modes: a CCP discharge used for the implantation step and a remote plasma (RP) discharge used for the selective removal. In their paper, Renaud et al. developed a two-step cycling process in this reactor consisting of an $\mathrm{H}_{2}$ implantation followed by $\mathrm{NH}_{3} / \mathrm{NF}_{3}$ remote plasma for the etching of LPCVD $\mathrm{SiN}$ films. It was shown that during the remote plasma step, the consumption of the silicon nitride proceeds through the formation of $\left(\mathrm{NH}_{4}\right)_{2} \mathrm{SiF}_{6}$ salts. When the plasma is stopped and the chamber pressure decreases back to the base pressure the salt are outgassed and the etch stops. When cycling the two steps, this outgassing happens between the removal step and the next implantation step leaving a pristine $\mathrm{SiN}$ at the end of each cycle. Promising results were obtained on blanket films with selectivity over non-modified silicon nitride and the underlying silicon however the process was not tested on patterned samples [4].

In this study we apply this two-step cycling process using the same reactor on PECVD nitride spacer. This silicon nitride has a richer concentration in hydrogen and is less plasma resistant than LPCVD films. The cycling will be carried out on patterned samples to prove the feasibility of the concept.

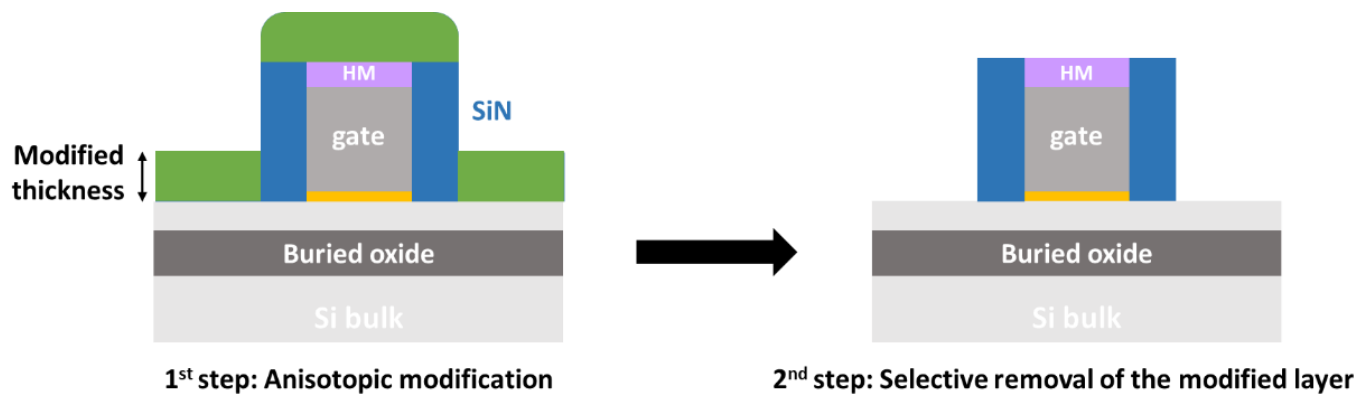

Figure 2. The smart etch concept applied on spacers.

\section{METHODS}

\subsection{Substrates}

The substrates used for this study are 75nm-thick SiN film deposited by PECVD on bare silicon that were patched on $300 \mathrm{~mm}$ silicon carrier wafers. Those wafers were used to understand the etching mechanisms involved in the two steps and develop the cycling process that has been applied to the patterned samples shown in figure 1a.

\subsection{Plasma reactor}

The plasma reactor used in this study is a 300mm prototype chamber presented in figure 3 . This reactor was well described by Renaud et al. [4]. Briefly, it includes a remote plasma source (RP) and a capacitively coupled plasma (CCP) source. The CCP unit is alimented by a RF power supply $(13,56 \mathrm{MHz})$ coupled to the bottom electrode on which the substrate lies. The electrostatic chuck is kept at $100^{\circ} \mathrm{C}$ for both steps, the walls are maintained at $80^{\circ} \mathrm{C}$ and the lid separating both units is at $130^{\circ} \mathrm{C}$. The ion implantation is carried out by flowing $\mathrm{H}_{2}$ at a pressure of $50 \mathrm{mT}$ Torr with a power of $50 \mathrm{~W}$ during $60 \mathrm{~s}$. In those conditions, the ion energy obtained is about $120 \mathrm{eV}$. The downstream plasma unit is alimented by a RF power supply $(13,56 \mathrm{MHz})$ separated from the CCP unit by showerheads that block the ions so that only the reactive neutrals reach 
the substrate. The selective removal of the modified layer uses a gas mixture of $\mathrm{He} / \mathrm{NH}_{3} / \mathrm{NF}_{3}$ at 2,5 Torr with a power of 250W [4].
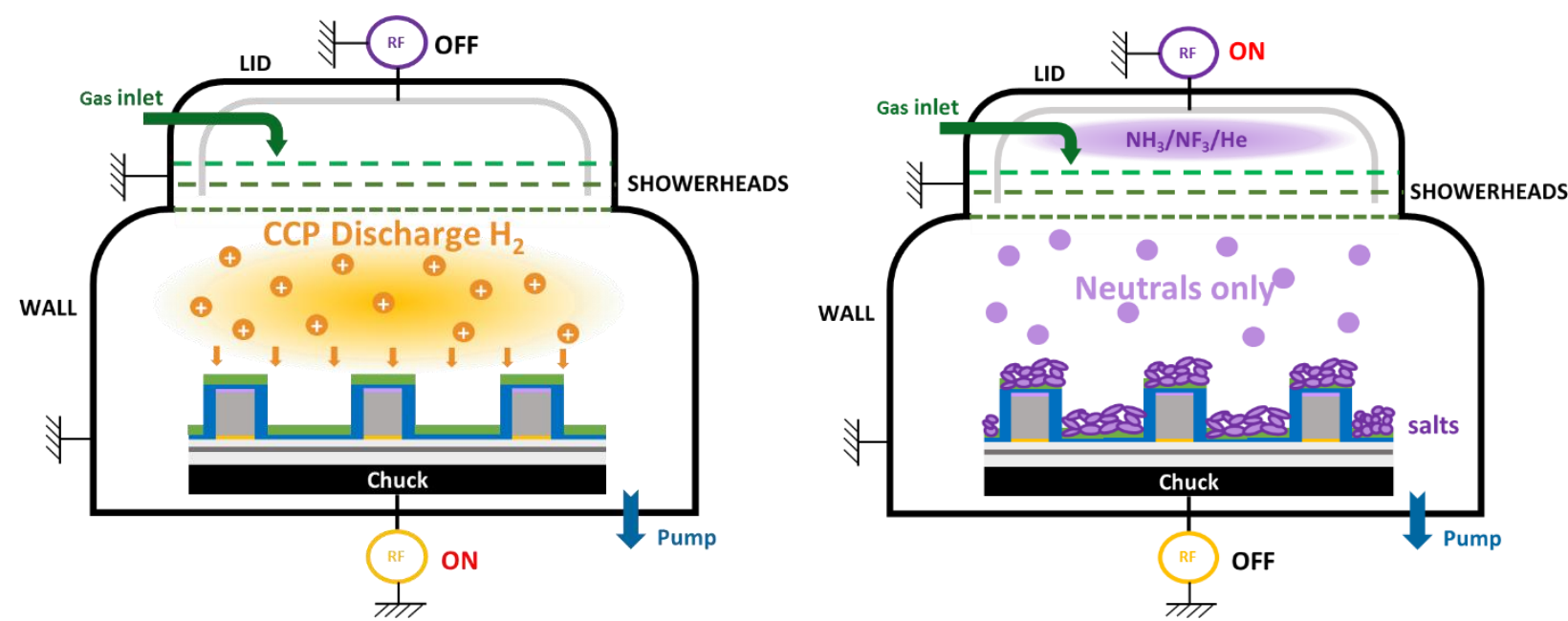

Figure 3. Schematics of the reactor used in this study. On the left during the implantation step, the $\mathrm{H}_{2}$ ions modify the surface. On the right during the remote plasma step, only the neutral species go through the showerheads and react with SiN to form salts.

\subsection{Characterizations}

The chemical composition of the surface before and after the implantation step is analyzed by angle resolved X-Ray photoelectron spectroscopy (AR-XPS) in quasi in-situ conditions thanks to a vacuum connection between the reactor and the XPS. The XPS is equipped with a high resolution monochromatic Al Ka X-ray source and a detector able to conduct angle resolved analysis between the angles of $23.75^{\circ}$ and $76.25^{\circ}$. This capability offers the possibility to reconstruct a depth profile of the atomic composition of the material surface. X-ray reflectometry (XRR) is also used to measure the thicknesses and densities of layers pre and post implantation. The etch kinetics of implanted and pristine SiN during the remote plasma step are monitored using a kinetic ellipsometer directly connected to the chamber. A model composed of the SiN layer and the salt layer is used to fit the ellipsometric data during the etch process [4]. Transmission electron microscopy (TEM) is performed to evaluate the spacer consumption and profile after etching.

\section{RESULTS AND DISCUSSION}

\subsection{Characterizations of the implantation step}

As shown by the XPS spectra in figure 4a) the pristine SiN film contains a Si-O [3] bond at 103.6eV indicating the presence of an oxidized layer at the surface. After a 60 s exposition to the hydrogen CCP discharge, the analysis reveals a modification of the surface with the formation of a new bond at $102.7 \mathrm{eV}$, labelled $\mathrm{SiN}^{*}$ that can be attributed to either $\mathrm{Si}-$ $\mathrm{F}$ [5] or Si-O-N [6] in figure 4b). As shown in figure 4c), Fluorine concentration is significantly increased by the implantation: although the CCP plasma is hydrogen based, the chamber's walls are contaminated with fluorine by other processes thus explaining the high amount post implantation. 
a) Pristine $\mathrm{SiN}$

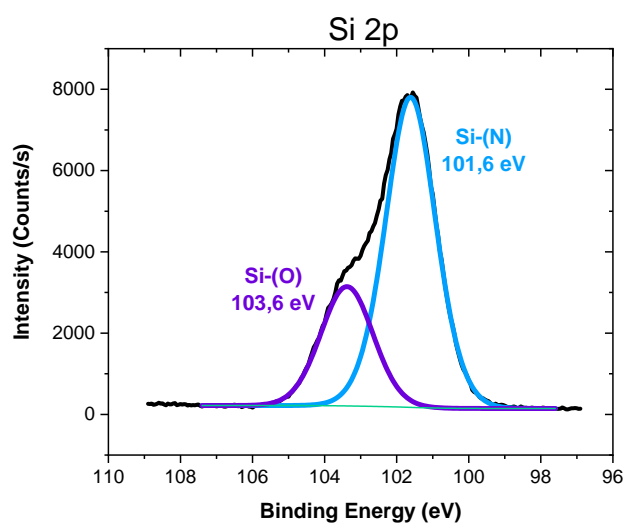

b) Post Implantation

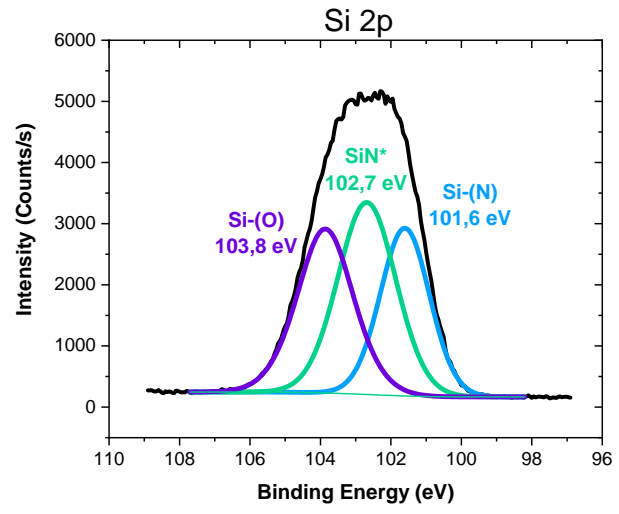

c)

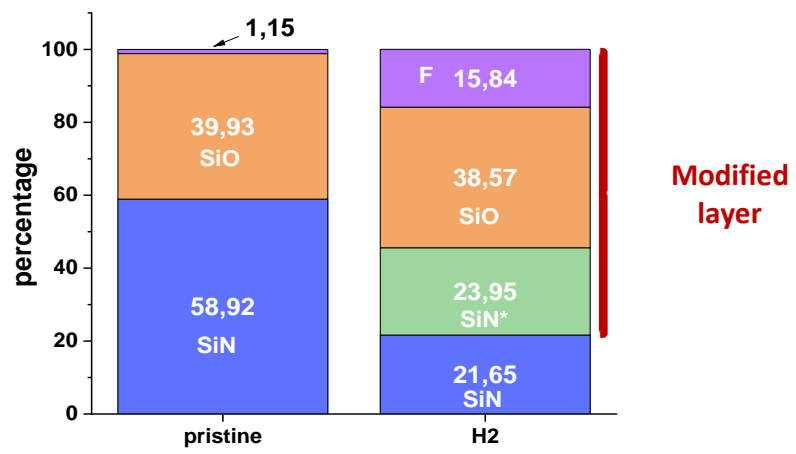

Figure 4. Deconvoluted XPS spectra of core level Si2p of SiN layer before (a) and after a $60 \mathrm{~s} \mathrm{(b)} \mathrm{H}_{2}$ plasma exposure, c) chemical composition of the $\mathrm{SiN}$ surfaces before and after $\mathrm{H}_{2}$ implantation ( $\mathrm{H}$ is not detected by XPS).

The surface density is also altered by the hydrogen CCP discharge. Indeed, the X-ray reflectometry results shown in figure 5a), shows that two layers are formed on top of the bulk of the film: a 3,6nm thick first layer with low density and an intermediate layer, closer in density to the bulk giving a total modified layer of $6 \mathrm{~nm}$. Thanks to the angular capability of our XPS, atomic concentration depth profile is reconstructed as shown in figure $5 \mathrm{~b}$ ). This analysis confirms the bilayer model predicted by the XRR. After the CCP discharge, Si-O bonds and fluorine are strongly present in the first $2 \mathrm{~nm}$ followed by an intermediate layer of $\mathrm{SiN}^{*}$ bonds from 2 to $4 \mathrm{~nm}$. Although the thicknesses given by AR-XPS does not match the one given by XRR, the profiles are qualitatively similar. Hydrogen cannot be detected by XPS but other studies have shown the presence of $\mathrm{SiH}$ and $\mathrm{NH}$ bonds in the whole modified depth [2] [4]. 


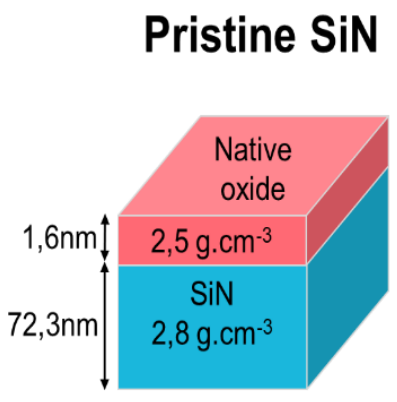

Total: $74 \mathrm{~nm}$

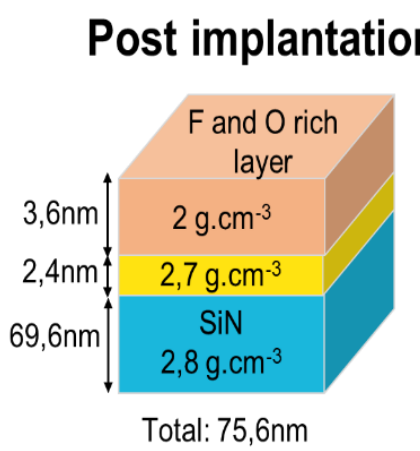

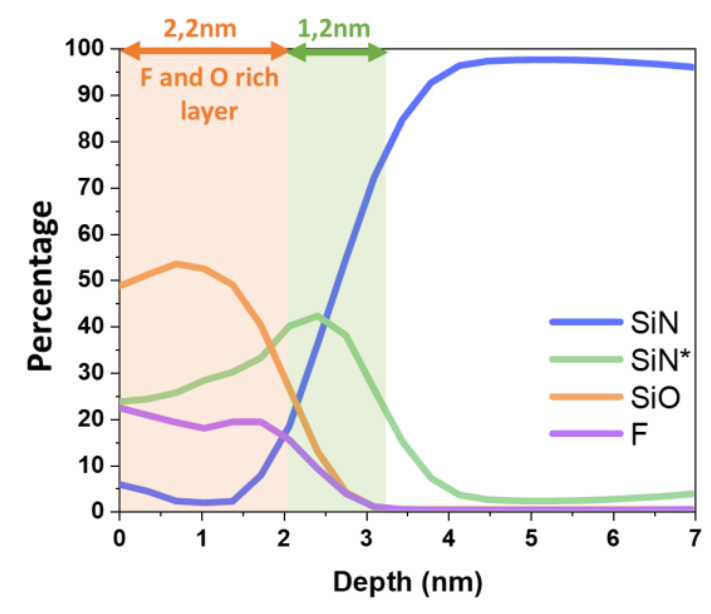

Figure 5. a) XRR results before and after implantation showing that the modified layer consists in a bilayer as a function of depth reconstructed by AR-XPS.

b) Atomic composition

To summarize, H2 implantation with the plasma conditions used in this study leads to a 6nm thick SiN modified layer. The top surface is enriched in $\mathrm{F}$ and $\mathrm{O}$ contaminants, and the modified layer density is lowered compare to the one of the pristine certainly because of $\mathrm{SiN}$ bond breaking and formation of $\mathrm{SiH}$ and $\mathrm{NH}$ bonds.

\subsection{Etch Kinetics of implanted and non-implanted SiN during RP plasma}

Figure 6 shows the etch kinetics of $\mathrm{SiN}$ films exposed to $\mathrm{He} / \mathrm{NH}_{3} / \mathrm{NF}_{3}$ remote plasma. Three samples are under investigation, a pristine $\mathrm{SiN}$ with its oxide, a deoxidized sample and a $\mathrm{SiN}$ that has been implanted with $\mathrm{H}_{2}$ (using the conditions described in section 2.2). First, it is observed that a deoxidized $\mathrm{SiN}$ film is not etched upon $\mathrm{NH}_{3} / \mathrm{NF}_{3} \mathrm{RP}$ exposure, while there is a 20s incubation time before the pristine $\mathrm{SiN}$ starts to ectc. This incubation time corresponds to the time needed for the neutral species to absorb and react. Renaud et al showed that the incubation time is due to a competition between the chemisorption of the reactive species that have physisorbed on the SiN surface and their desorption. Both mechanisms are very sensitive to the substrate temperature and surface state. An increase in temperature tends to favor the desorption of the reactants rather than the chemisorption leading to an increase of the incubation times and a slowing down of the etching kinetics. Two hypothesis could explain the different etch behavior of pristine compared to deoxidized SiN: either the presence of an oxide layer, and presumably $\mathrm{OH}$ terminated bonds increases the residence time of the reactive neutrals in the physisorbed state on the SiN surface, increasing thus the probability to subsequently chemisorb, or it decreases the activation energy of the chemisorption step. After the hydrogen implantation of the pristine $\mathrm{SiN}$, the reaction begins as soon as the remote plasma starts which means that the modification of the surface induced by the implantation activates the physisorption and chemisorption kinetics. We believe that several factors are responsible for the etch catalysis: the incorporation of $\mathrm{O}$ and $\mathrm{F}$ during the implantation, the dangling bonds generated by the ion bombardment, and the H-terminated bonds that are formed. While the implantation step provides etch activation it does not significantly modifies the etch rate in the stationary regime, contrary to what was found on LPCVD spacers where a higher etch rate was observed on the implanted silicon nitride [4]. However, on PECVD silicon nitride the activation of the etch offers a process window of infinite selectivity between the implanted and non-implanted surfaces. As it will be discussed in section 3.3, this result can be exploited for the development of spacer etching process whose aim is to selectively remove the horizontal part of the film deposited on a pattern over the vertical parts. 


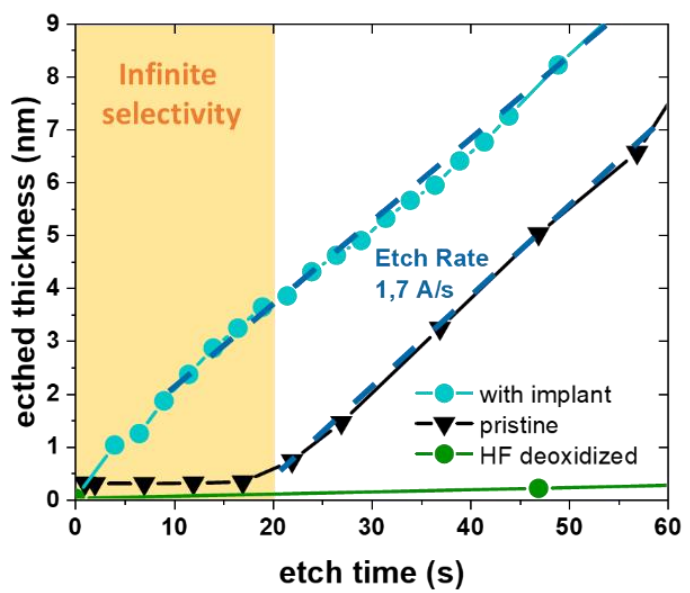

Figure 6. Etch kinetics of implanted (blue), non-implanted (black) and deoxidized SiN (green) when exposed to a remote plasma of $\mathrm{He} / \mathrm{NH}_{3} / \mathrm{NF}_{3}$ measured by in situ kinetic ellipsometry.

As the SiN surface state is the key for etch ignition during the RP process, we have investigated other plasma chemistries for the implantation step, including $\mathrm{O}_{2}$ and $\mathrm{NH}_{3}$. Oxygen and $\mathrm{NH}_{3}$ based CCP discharges were carried out in conditions ensuring ion energy of about $120 \mathrm{eV}$, similarly to $\mathrm{H}_{2}$ implantation. The chemical composition of each sample after implantation obtained by XPS is shown in figure 7b). Every type of implantation adds fluorine to the surface due to the chamber contamination although the $\mathrm{NH}_{3}$ based implant limits the contamination to $7 \%$ half less than the contamination given by the $\mathrm{H}_{2}$ plasma. The Oxygen implant does not form $\mathrm{SiN}^{*}$ bonds but oxidize the surface. The $\mathrm{NH}_{3}$ implantation seems to implant some nitrogen in the surface as the total concentration of $\mathrm{SiN}$ and $\mathrm{SiN}^{*}$ bonds reach about $85 \%$ which is more than the pristine sample. Those modifications of the surface give various etch kinetics during the remote plasma step, as shown in figure 7a). The $\mathrm{NH}_{3}$ implant gives the same kinetics and incubation time as the $\mathrm{H}_{2}$ implant while the oxygen implanted film behaves like a pristine film. These results suggest that H-terminated bonds are mainly responsible for the etch activation

a)

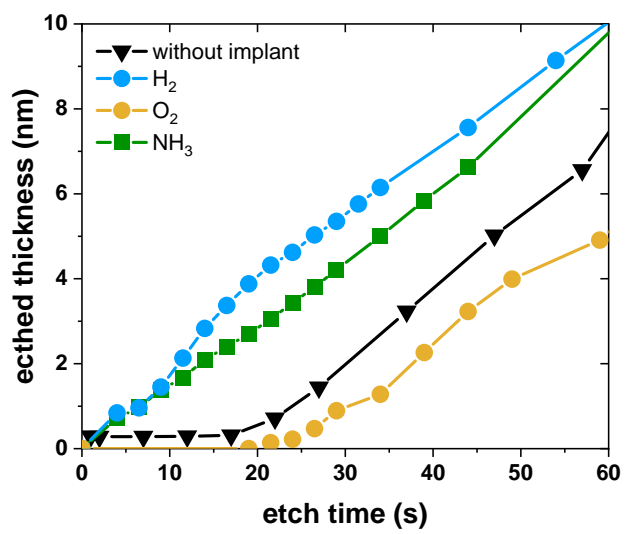

b)

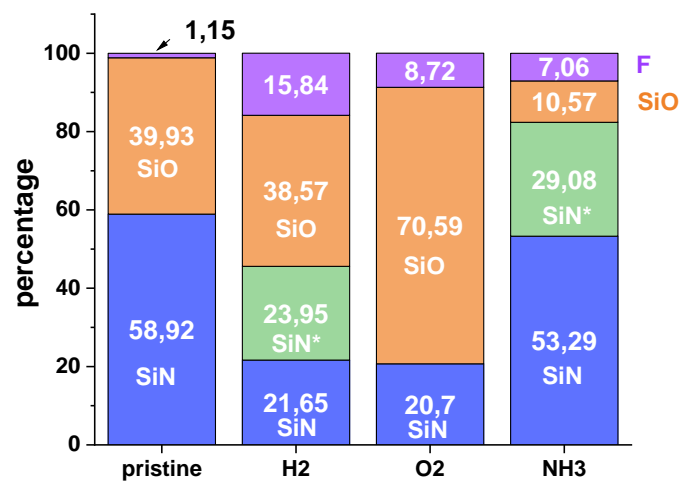

Figure 7. a) Etch kinetics in the remote plasma process according to the plasma chemistry used for the implantation. b) Chemical composition of the surface obtained by XPS analysis. 
In the rest of this study the $\mathrm{H}_{2}$ discharge will be used as it offers the best process window between implanted and nonimplanted surfaces.

\subsection{Cycling Results}

Based on the results of figure 6, we develop a two-step cycling process composed of the $\mathrm{H}_{2}$ implantation step followed by the $\mathrm{NH}_{3} / \mathrm{NF}_{3}$ remote step whose processing time is adjusted to be in the infinite process window, namely under 20 s so that the non-implanted surfaces remain unetched. In the following, a $60 \mathrm{~s} \mathrm{H}_{2} \mathrm{CCP}$ discharge and a $15 \mathrm{~s} \mathrm{RP}$ step have been cycled successively. Etched $\mathrm{SiN}$ and salts thicknesses at each cycle have been measured and are represented in figure $8 \mathrm{a}$ ). The resulting etched thickness at each cycle has two components: the etch due to the sputtering during the first step of CCP discharge and the etch due to the remote plasma removal step. The kinetics results shown in figure 8a) indicates that around $20 \mathrm{~nm}$ of salts are produced during these $15 \mathrm{~s}$ of remote plasma. The implantation step is achieved at a pressure chamber of $50 \mathrm{mT}$ orr chamber while the remote plasma step operates at 2.5Torr. There is a decrease in pressure between the remote plasma step and the implant step, which allows the salt to outgas during this period, meaning that the SiN surface is salt free at each new cycle. The first loop etches around $1,5 \mathrm{~nm}$ of the material while the other loops etch around $5 \mathrm{~nm}$ in total. The first $1,5 \mathrm{~nm}$ etched corresponds to the oxidized layer present on top of the bulk of the SiN, this layer seems to etch slower than the others and is less sensitive to the sputtering effect of the $\mathrm{H}_{2}$ plasma. Once the oxidized layer is removed, each loop is very similar to the next one, proving the high repeatability of the process on the bulk SiN film.

a)

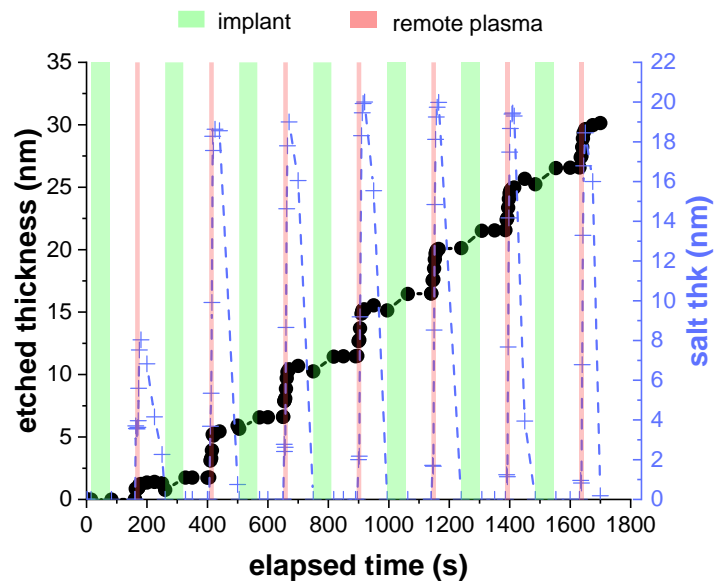

b)

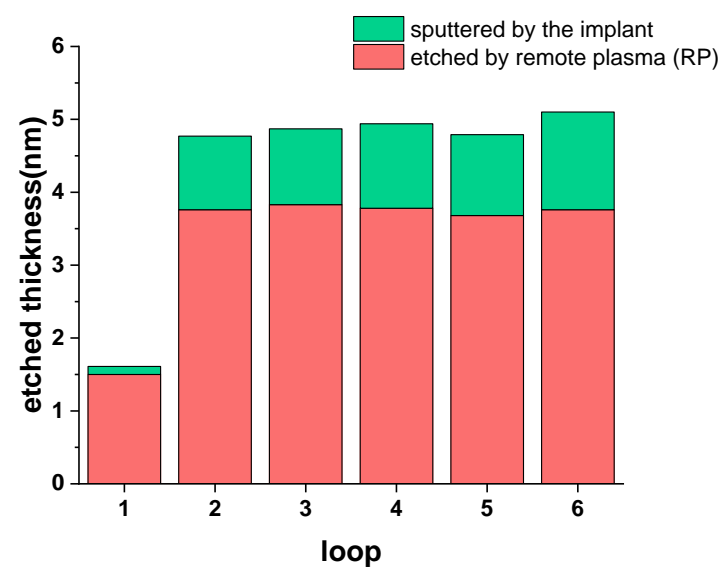

Figure 8. a) Etch kinetics of the sample while cycling the two steps: in black is the etched thickness of silicon nitride, in blue the salt thickness formed during the remote plasma step. b) Summary of the etched thicknesses for each cycle during the implant step (green) and the remote plasma step (red).

Following those promising results, the cycling of the two steps has been performed on patterned sample as shown in the TEM images in figure 9. An eight cycles process was applied to the sample which had a $45 \mathrm{~nm}$-thick SiN layer deposited by PECVD. After eight cycles, the process gives a promising spacer profile with a vertical shape and minimal lateral consumption compared to the conventional process shown in figure 1. 

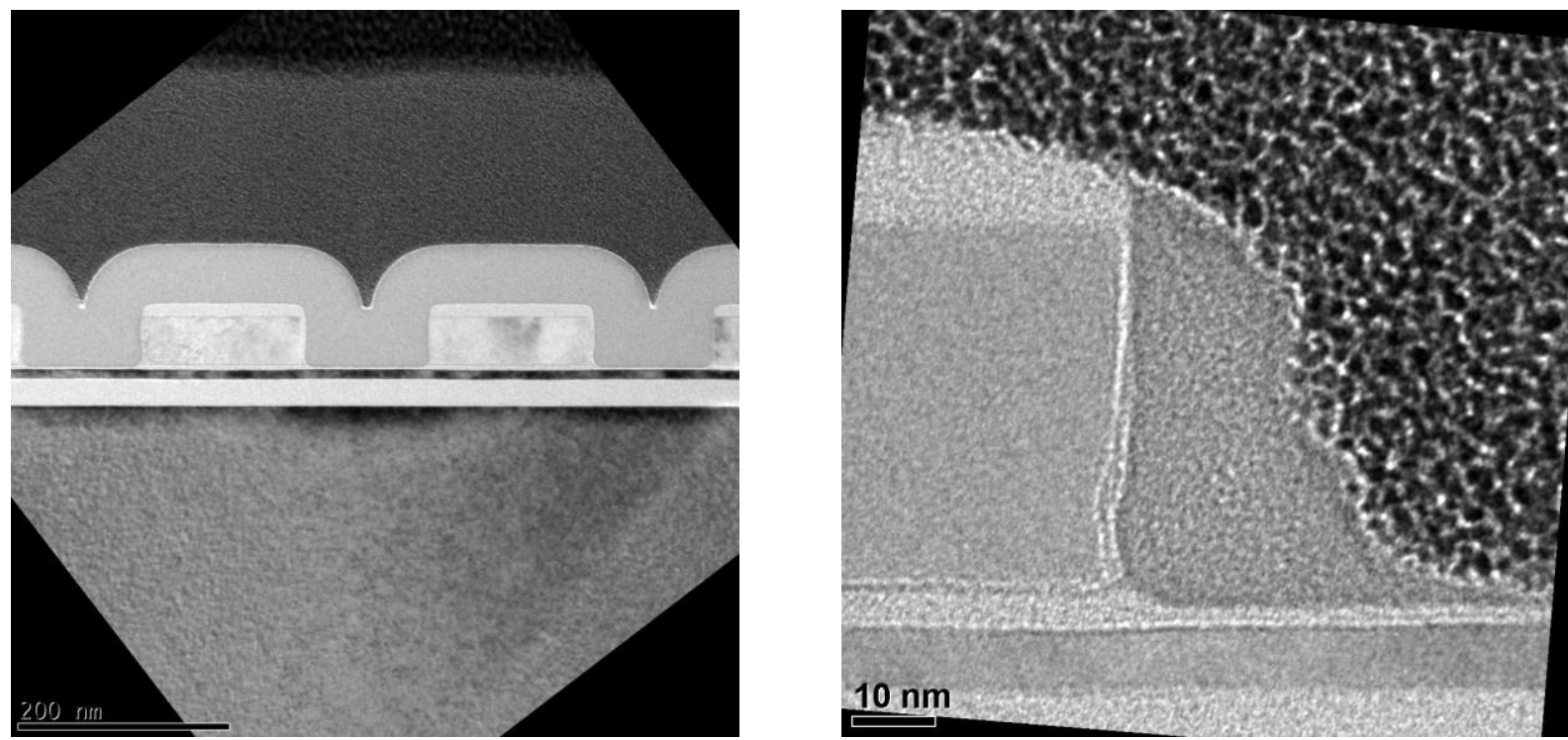

Figure 9. TEM pictures before and after 8 cycles of implant/removal

\section{CONCLUSION}

The two-step cycling process proposed in this study presents interesting pathways to etch PECVD silicon nitride spacers layers with a high precision while being anisotropic. This study shows that the implantation step modifies the SiN surface state and subsequently activates the etch when exposed to the remote $\mathrm{NH}_{3} / \mathrm{NF}_{3}$ plasma step. Kinetic ellipsometry showed that implantation performed with hydrogen plasma is the most effective to activate the etch and is thus the only one so far providing a process window with infinite selectivity. Cycling the two steps in this process window proves itself very repeatable past the etch of the native oxide of the silicon nitride films with an etched thickness of around $5 \mathrm{~nm}$ per cycle. First experiments on patterned samples show promising results, ongoing researches are currently focusing on the development of this process on patterned samples by coupling conventional etching process with the cycling approach as well as understanding the mechanism of the etch activation better.

\section{ACKNOWLEDGEMENT}

This work was partly supported by the french RENATECH network.

\section{REFERENCES}

[1] N. Posseme, O. Pollet, and S. Barnola, “Alternative process for thin layer etching: Application to nitride spacer etching stopping on silicon germanium”, Appl. Phys. Lett. 105, 051605 (2014).

[2] O. Pollet, N. Posseme, V. Ah-Leung, M. Garcia Barros, "Thin Layer Etching of Silicon Nitride: Comparison of Downstream Plasma, Liquid HF and Gaseous HF Processes for Selective Removal After Light Ion Implantation”, Solid State Phenomena 255:69-74 (2016).

[3] H. Ogawa, T. Arai, M. Yanagisawa, T. Ichiki, and Y. Horiike, "Dry Cleaning Technology for Removal of Silicon Native Oxide Employing Hot NH3/NF3 Exposure”, Jpn. J. Appl. Phys., 41, p. 5349 (2002). 
[4] V.Renaud, C. Petit-Etienne, J. Barnes, J. Bisserier, O. Joubert, E. Pargon, J. Appl. Phys. 126, 243301 (2019).

[5]. B.S. Sahu, A. Kapoor, P. Srivastava, O.P. Agnihotri and S.M. Shivaprasad, "Study of thermally grown and photoCVD deposited silicon oxide-silicon nitride stack layers", Semiconductor Science and Technology, Volume 18, Number 7 (2003).

[6] Sunghoon Lee, Seodaemun-gu, Jinho Oh, Kyumin Lee, and Hyunchul Sohn "Ultrahigh selective etching of Si3N4 films over SiO2 films for silicon nitride gate spacer etching”, Journal of Vacuum Science \& Technology B 28, 131 (2010). 